\section{Detection and Characterization of New Coronavirus in Bottlenose Dolphin, United States, 2019}

\author{
Leyi Wang, Carol Maddox, Karen Terio, \\ Saraswathi Lanka, Richard Fredrickson, \\ Brittany Novick, Celeste Parry, Abby McClain, \\ Kyle Ross
}

\begin{abstract}
Author affiliations: University of Illinois, Urbana, Illinois, USA
(L. Wang, S. Lanka, R. Fredrickson); University of Illinois College of Veterinary Medicine, Urbana (C. Maddox); University of Illinois College of Veterinary Medicine, Brookfield, Illinois, USA (K. Terio); National Marine Mammal Foundation, San Diego, California, USA (B. Novick, C. Parry, A. McClain); Naval Information Warfare Center Pacific, San Diego (K. Ross)
\end{abstract}

DOI: https://doi.org/10.320/eid2607.200093

We characterized novel coronaviruses detected in US bottlenose dolphins (BdCoVs) with diarrhea. These viruses are closely related to the other 2 known cetacean coronaviruses, Hong Kong BdCoV and beluga whale CoV. A deletion in the spike gene and insertions in the membrane gene and untranslated regions were found in US BdCoVs (unrelated to severe acture respiratory syndrome coronavirus 2 ).

The coronavirus family consists of single-stranded, 1 positive-sense RNA viruses that cause respiratory, gastrointestinal, hepatic, and neurologic diseases of different host species. On the basis of genetic characterization, coronaviruses have been classified into 4 genera: Alphacoronavirus, Betacoronavirus, Gammacoronavirus, and Deltacoronavirus. Cetacean coronavirus is a recently proposed new species in the genus Gammacoronavirus, in addition to a common species (Avian coronavirus) (1). Cetacean coronavirus species contains bottlenose dolphin coronavirus (BdCoV) HKU22, identified in 2014, and beluga whale coronavirus (BWCoV) SW1, identified in 2008 (1,2). We report detection and genetic characterization of $\mathrm{BdCoV}$ in bottlenose dolphins in the United States; all dolphins had clinical signs consistent with gastrointestinal discomfort.

Four Atlantic bottlenose dolphins cared for by the US Navy Marine Mammal Program (San Diego, CA) showed development of an acute onset of clinical illness with clinical signs consisting of inappetence $(n=4)$, diarrhea $(n=3)$, and lethargy $(n=2)$ during April and May 2019. We collected fecal samples as part of the minimum workup for acute illness. Among all viruses we tested by using conventional PCRs, only coronavirus showed a positive result for all 4 dolphins.

We further evaluated samples by using next-generation sequencing as described (3). De novo assembly analysis of raw FASTQ data showed that 4 near complete genomes of $\mathrm{BdCoV}$ were assembled. Gaps were closed by Sanger sequencing at ACGT, Inc. (https:// www.acgtinc.com). The genomes of all 4 US BdCoVs (37112-1, -2, -3, and -4) comprised 31,728 nt (GenBank accession nos. MN690608-11), which were shorter than those of 3 Hong Kong BdCoVs (HKBdCoVs) (31,750-31,758 nt).

Further analysis of all individual genes showed that the 4 US BdCoV strains showed similar identities to both $\mathrm{HK}-\mathrm{BdCoVs}$ and $\mathrm{BWCoV}$ in open reading frame (ORF) 1a, ORF1b, nonstructural (NS) 7, NS8, NS9, and NS10. However, US BdCoV strains showed higher identities to HK-BdCoVs than to BWCoV only in spike (S), envelope (E), membrane (M), and NS5a instead of all remaining genes (Appendix Table, Figure 1, https:/ / wwwnc. cdc.gov/EID/article/26/7/20-0093-App1.pdf).

The 4 US BdCoV strains showed relatively higher identities to BWCoV than to HK-BdCoVs in NS5b $(95.9 \%$ vs. $93.8 \%-94.0 \%)$, NS5c $(98.4 \%$ vs. $97.7 \%-$ 97.9\%), NS6 (94.9\% vs. $88.6 \%-88.9 \%)$, and nucleocapsid protein (97.9\% vs. $96.1 \%-96.5 \%)$ genes. Analysis of amino acid identities of different ORFs also showed similar patterns (Appendix Table).

Phylogenetic analysis of complete genomes showed that the 4 US BdCoVs were clustered with 3 HK-BdCoVs strains and distantly related to $\mathrm{BdCoV}$ SW1 but were distinct from avian coronaviruses (Figure). Phylogenetic trees for individual genes showed that US BdCoV strains have a greater correlation with HK-BdCoVs than BWCoV in the S, E, M, NS5a, and NS7 genes and to BWCoV than $\mathrm{HK}-\mathrm{BdCoVs}$ in remaining genes (Figure; Appendix Figures 2, 3).

Compared with the Hong Kong CF090331 strain, all US BdCoV strains have a 42-nt deletion in the S1 region at positions $21366-21407$ encoding an S protein that is 14-aa shorter (Appendix Figure 4). Compared with $3 \mathrm{HK}-\mathrm{BdCoVs}$ strains, a 3-nt insertion (ACA) at positions 25417-25419 was found in the M gene of the 4 US BdCoVs, leading to a frameshift mutation in the $M$ protein that was 1 amino acid longer (Appendix Figure 5).

In addition, a 4-nt insertion (TATA) was found in the $5^{\prime}$ untranslated region (UTR) of US BdCoV strains, and a 1-nt insertion (T) was found in the $3^{\prime}$ UTR of US $\mathrm{BdCoV}$ strains (Appendix Figure 6). Similar to findings of a previous report (1), because of 1 nt mutation $(\mathrm{G} \rightarrow \mathrm{T}$ at position 28268) of the US BdCoV strains, a premature stop codon in the NS7 gene resulted in 

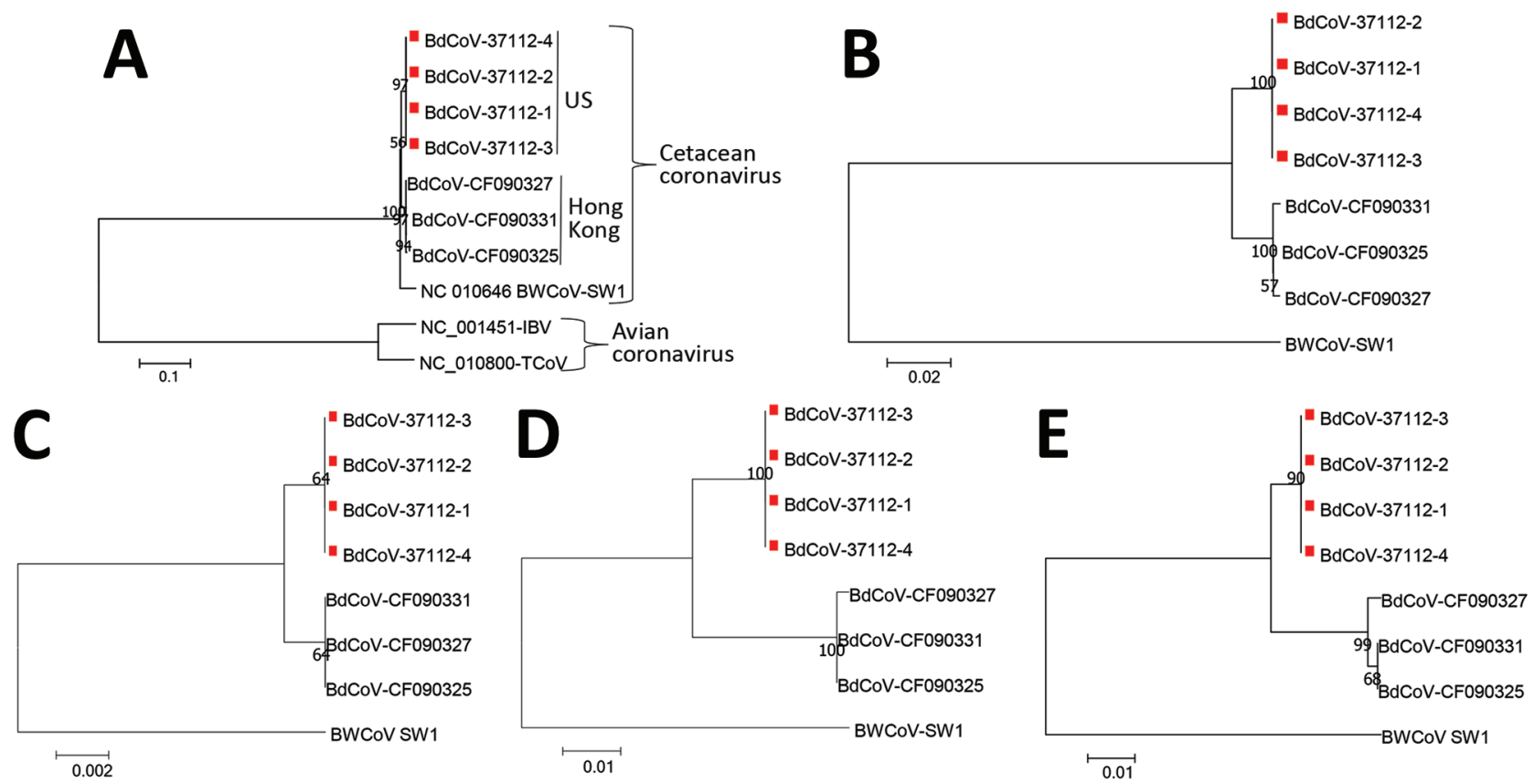

Figure. Phylogenetic analysis of A) complete genome, B) spike, C) envelope, D) matrix, and E) nonstructural protein 5a genes of gammacoronaviruses, including 4 US BdCoVs, 37112-1 to -4 (GenBank accession nos. MN690608-MN690611, indicated with red squares); 3 Hong Kong BdCoVs (accession nos.: CF090327, KF793825; CF090331, KF793826; CF090325, KF793824); and 1 BWCoV (SW1, accession no. NC_010646). Numbers along branches are bootstrap values. Scale bars indicate nucleotide substitutions per site. $\mathrm{BdCoV}$, bottlenose dolphin coronavirus; BWCoV, beluga whale coronavirus.

an NS7a (42 aa) and an NS7b (117 aa). The position of the premature stop codon in the US BdCoVs was different from that for $2 \mathrm{HK}-\mathrm{BdCoVs}$ (CF090325 and CF090331), which encode different sizes of NS7a (63 aa) and NS7b (34 aa) proteins.

$S$ deletion variants are commonly observed in coronaviruses. Porcine respiratory coronavirus is an $S$ gene deletion mutant of transmissible gastroenteritis virus and causes a respiratory disease instead of gastroenteritis in pigs because of a large deletion $(>600$ $\mathrm{nt}$ ) in the $\mathrm{N}$ terminal of the $\mathrm{S}$ gene (4). In addition, a large deletion (591 nt) in S1 resulted in a change in virulence of porcine epidemic diarrhea virus (5). In our study, we observed that $4 \mathrm{US}$ BdCoVs had a 42-nt deletion in the S1 gene. It is unclear whether this deletion region is related to the viral tropism and virulence, and warrants further studies.

During a surveillance study in Hong Kong, China, BdCoV was identified only in fecal samples from dolphins that had no notable clinical signs (1). In our study, genetically related $\mathrm{BdCoVs}$ were detected in dolphins that had diarrhea, lethargy, and inappetence in the United States. It is possible that unique genetic features of US BdCoVs, including a sequence deletion in the $S$ gene and an insertion in the $M$ gene and $5^{\prime}$ and 3' UTRs, and mutations in different genes might have contributed to the observed clinical diarrhea signs in US dolphins. Additional surveillance is needed to monitor the evolution of this virus worldwide.

\section{About the Author}

Dr. Wang is a clinical assistant professor at the College of Veterinary Medicine, University of Illinois, Urbana. His research interests are diagnosis of viral infectious diseases and novel pathogen discovery.

\section{References}

1. Woo PC, Lau SK, Lam CS, Tsang AK, Hui SW, Fan RY, et al. Discovery of a novel bottlenose dolphin coronavirus reveals a distinct species of marine mammal coronavirus in Gammacoronavirus. J Virol. 2014;88:1318-31. https:/ / doi.org/10.1128/JVI.02351-13

2. Mihindukulasuriya KA, Wu G, St Leger J, Nordhausen RW, Wang D. Identification of a novel coronavirus from a beluga whale by using a panviral microarray. J Virol. 2008;82:50848. https:/ / doi.org/10.1128/JVI.02722-07

3. Wang L, Stuber T, Camp P, Robbe-Austerman S, Zhang Y. Whole-genome sequencing of porcine epidemic diarrhea virus by Illumina MiSeq platform. In: Wang L, editor. Animal coronaviruses. Springer protocols handbooks. Totowa (NJ): Humana Press; 2016. p. 201-8.

4. Wang L, Zhang Y. Genomic characterization of a new PRCV variant, United States, 2014. Transbound Emerg Dis. 2017;64:672-4. https://doi.org/10.1111/tbed.12400

5. Hou Y, Lin CM, Yokoyama M, Yount BL, Marthaler D, 
Douglas AL, et al. Deletion of a 197-amino-acid region in the N-terminal domain of spike protein attenuates porcine epidemic diarrhea virus in piglets. J Virol. 2017;91:e00227-17. https:/ /doi.org/10.1128/JVI.00227-17

Address for correspondence: Leyi Wang, Department of Veterinary Clinical Medicine and the Veterinary Diagnostic Laboratory, College of Veterinary Medicine, University of Illinois, Urbana, IL, 61802, USA; email: leyiwang@illinois.edu

\section{Human Case of Severe Fever with Thrombocytopenia Syndrome Virus Infection, Taiwan, 2019}

\author{
Shih-Huan Peng, ${ }^{1}$ Su-Lin Yang, ${ }^{1}$ Shih-En Tang, \\ Tzy-Chen Wang, Tung-Chien Hsu, Chien-Ling Su, \\ Meng-Yu Chen, Masayuki Shimojima, \\ Tomoki Yoshikawa, Pei-Yun Shu
}

\begin{abstract}
Author affiliations: Centers for Disease Control, Ministry of Health and Welfare, Taipei, Taiwan (S.-H. Peng, S.-L. Yang, T.-C. Wang, T.-C. Hsu, C.-L. Su, M.-Y. Chen, P.-Y. Shu); Tri-Service General Hospital, Taipei (S.-E. Tang); National Defense Medical Center, Taipei (S.-E. Tang); Institute of Aerospace and Undersea Medicine, National Defense Medical Center, Taipei (S.-E. Tang); National Institute of Infectious Diseases, Tokyo, Japan (M. Shimojima, T. Yoshikawa)
\end{abstract}

DOI: https://doi.org/10.3201/eid2607.200104

We report on a 70-year-old man with fever, leukopenia, thrombocytopenia, vomiting, malaise, dyspnea, and consciousness disturbance who was infected with severe fever with thrombocytopenia syndrome virus in northern Taiwan, 2019. This autochthonous case was confirmed by reverse transcription PCR, virus isolation, and genomic sequencing.

Severe fever with thrombocytopenia syndrome (SFTS) is a tickborne infection caused by the SFTS virus (SFTSV, also known as Huaiyangshan banyangvirus), which was identified in China in 2009 (1) and afterward in South Korea (2), Japan (3), and Vietnam

${ }^{1}$ These first authors contributed equally to this article.
(4). Since then, the number of SFTS cases in East Asia has risen rapidly. Therefore, laboratory-based surveillance of SFTS has been conducted in the routine molecular diagnosis of arboviral infections in the Taiwan Centers for Disease Control (Taiwan CDC) since 2013. We identified a patient in Taiwan with laboratory-confirmed SFTS who was originally suspected of having dengue or rickettsial infections.

In November 2019, a 70-year-old man who lived in northern Taiwan and had no travel history was admitted to the hospital with a 9-day history of fever $\left(38.8^{\circ} \mathrm{C}-39.2^{\circ} \mathrm{C}\right)$, chills, nausea, vomiting, and malaise. The patient had underlying hypertension and type 2 diabetes mellitus that was controlled without medication. At hospital admission, we noted a generalized rash over the trunk and both feet. Laboratory examinations showed that the patient had leukopenia; thrombocytopenia; abnormal prothrombin time; elevated levels of aspartate transaminase, alanine transaminase, creatinine kinase, and C-reactive protein; and diagnostic disseminated intravascular coagulation (Table). Chest radiography and chest computed tomography showed patchy consolidations and ground-glass opacities of both lungs. A few hours after admission, the patient experienced a general tonic-clonic seizure, with worsening consciousness and dyspnea. He was transferred to the intensive care unit, where intubation and ventilator support began. He also received massive blood transfusions for severe thrombocytopenia, active mucosal (oral, nasal, and gastrointestinal tract) bleeding, and disseminated intravascular coagulation. Blood and sputum cultures revealed that the patient was infected with Pseudomonas aeruginosa; he received piperacillin/ tazobactam, doxycycline, and clarithromycin as empirical therapy. Results of laboratory tests for hepatitis $\mathrm{A}$ and $\mathrm{B}$ viruses, cytomegalovirus, herpes simplex virus, adenovirus, and influenza were all negative. After the patient received a diagnosis of SFTSV infection, he received treatment with intravenous immunoglobulin for 5 days. However, his condition continued to deteriorate progressively. The patient died on day 40 after illness onset as a result of multiorgan failure. Delayed diagnosis and the presence of underlying conditions in this patient, including hypertension and diabetes mellitus, may be associated with his severe disease and death (5).

The patient often spent time on a vegetable farm in a mountainous area without wearing shoes, raising suspicions for arboviral and rickettsial infections. The hospital sent blood samples, collected from the patient before the blood transfusions on day 12 after illness onset, to the Taiwan CDC for 